\title{
LIMITACIONES PARA EL ESTABLECIMIENTO DE PLÁNTULAS EN ARENALES DE ORIGEN VOLCÁNICO
}

\author{
Irma Jannetth Nava-Sosa ${ }^{1}$, Roberto Lindig-Cisneros ${ }^{2}$, Ek del-Val ${ }^{2}$ y Sabina Irene Lara-Cabrera ${ }^{1,3}$ \\ 'Laboratorio de Sistemática Molecular de Plantas, Facultad de Biología, \\ Universidad Michoacana de San Nicolás de Hidalgo \\ ${ }^{2}$ Centro de Investigaciones en Ecosistemas, Universidad Nacional Autónoma de México \\ ${ }^{3}$ Autor para la correspondencia: slaracabrera@gmail.com
}

\begin{abstract}
Resumen: La dispersión de semillas, la disponibilidad de micrositios y la herbivoría pueden afectar severamente el resultado de las actividades de restauración ecológica. En este estudio se evaluó el papel de estos tres factores en el establecimiento de plántulas en arenales de origen volcánico. Se evaluó el efecto de exclusión de mamíferos pequeños mediante un cercado (presencia/ausencia) y diferencias en micrositios mediante tres tratamientos de acolchado (control, $227 \mathrm{~g} / \mathrm{m}^{2}$ y $576 \mathrm{~g} / \mathrm{m}^{2}$ ), en parcelas colocadas al azar a diferentes distancias del borde del bosque. Los resultados indican que el efecto más importante es la distancia del borde del bosque, siendo significativo para el total de plántulas $\left(F_{(132,1)}=69, P<0.0001\right)$ y para 10 especies de 28 de manera individual. El efecto del cercado fue significativo sólo para especies de Cyperus y el acolchado sólo a través de la interacción con las dos variables anteriores. Los resultados sugieren que la dispersión, no la herbivoría, es la principal limitante para el establecimiento de plántulas en los arenales.
\end{abstract}

Palabras clave: restauración ecológica, tefra, dispersión, exclusión, acolchado

\begin{abstract}
Seed dispersal, microsite availability, and herbivory have a considerable effect on the outcome of ecological restoration efforts. In this study we evaluated the role of these factors on the establishment of seedlings in a sand deposit of volcanic origin. The role of fencing (presence/absence) as exclusion for small mammals, and three mulching treatments (control, 227g/ $\mathrm{m}^{2}$ and 576g/ $\mathrm{m}^{2}$ ), for creating differences in microsites, in plots placed randomly at different distances from the forest edge. Results show that the most important factor is distance from the forest edge being significant for all seedlings regardless of species was evaluated $\left(F_{(132,1)}\right.$ $=69, P<0.0001$ ), and independently for 10 out of 28 species. The exclusions were significant only for establishment of plants of Cyperus spp, and mulching only through interactions. Results suggest that seed dispersal, and not herbivory, is the main limiting factor for seedling establishment in sand deposits.
\end{abstract}

Key words: ecological restoration, tephra, dispersion, exclusion, mulching

a Comunidad Indígena de Nuevo San Juan ParangaricuLtiro (CINSJP), es reconocida a nivel internacional por el manejo sustentable de sus bosques, y debido a que de ellos obtienen la materia prima para su empresa (madera y resina) tienen interés en restaurar las áreas desprovistas de vegetación, como son los arenales (Blanco-García y LindigCisneros, 2005). Los arenales se formaron en áreas sin un dosel arbóreo al depositarse ceniza del volcán Paricutín que entró en erupción en 1943 y se mantuvo activo por nueve años (Delgado-Granados, 2001) . Los estudios del desarrollo de la vegetación en las zonas afectadas por la erupción de volcanes han mostrado un incremento en el número de especies bajo diferentes condiciones, en particular en sus conos, los derrames de lava y los bosques que fueron afectados por la lluvia de ceniza volcánica (Eggler, 1959, 1963; Rejmánek et al., 1982). Sin embargo, en los arenales los procesos sucesionales no han permitido el establecimiento de especies arbóreas de manera natural y por lo tanto predomina la vegetación herbácea y arbustiva que forma manchones rodeados de arena desnuda (Lindig-Cisneros et al., 2006). A pesar de esto, las especies arbóreas se establecen si son sembradas artificialmente y si se toman otras medidas para su protección, como por ejemplo el uso de acolchados (Blanco-García y Lindig-Cisneros, 2005), aunque la vege- 
tación del sotobosque no se establece aún cuando ya está presente una cobertura arbórea.

Estudios previos en la misma zona sugieren que la profundidad de la ceniza volcánica es un factor limitante que tiene un efecto diferencial en el establecimiento de las plantas, de tal forma que la distribución espacial de las plantas en los arenales parece estar relacionada con la profundidad de la arena (Gómez-Romero et al., 2006). Otros factores que limitan o afectan el establecimiento de diferentes especies de plantas nativas en el arenal son las temperaturas extremas del sustrato, debido al color obscuro de la ceniza volcánica, y en el caso de algunas especies arbustivas, como por ejemplo Lupinus elegans Kunth, la herbivoría también es un factor importante (Blanco-García y Lindig-Cisneros, 2005). Para esta especie, los daños por herbivoría en el sitio son similares a los que se han reportado en otras experiencias de restauración en donde la herbivoría causó pérdidas hasta del 80\% para especies arbóreas (Holl y Quiros-Nietzen, 1999; Bonfil, 1998). En los experimentos llevados a cabo en arenales de la CINSJP la herbivoría fue particularmente intensa en época de lluvias, durante los meses de agosto, septiembre y octubre (Blanco-García, 2005), a diferencia de otros sitios en donde la mayor incidencia de herbivoría ocurre en época de secas (Bonfil et al,. 2000). Adicionalmente, en el caso de Lupinus elegans, la talla determinó la susceptibilidad a la herbivoría, ya que los individuos de menor tamaño fueron los más consumidos (Blanco-García, 2005), lo que coincide con otros estudios (Rodríguez de la Vega, 2003). Lo anterior sugiere que la herbivoría de plántulas puede ser también un factor determinante para impedir el establecimiento de la vegetación en sitios con cenizas volcánicas. El efecto de la herbivoría podría ser fácilmente determinado al controlar el acceso a herbívoros mediante la colocación de cercas. Se ha documentado que el crecimiento y/o supervivencia de las plántulas pueden ser incrementados significativamente por la colocación de cercas (Shelton y Intuye, 1995; Case y Kauffman, 1997; Opperman y Merenlender, 2000; Sweeney et al., 2002; Frankland y Nelson, 2003; del-Val y Crawley, 2005; del-Val et al., 2007; Howe, 2008).

La CINSJP ha eliminado la capa de ceniza volcánica en varios arenales como una medida para facilitar la restauración ecológica de estos sitios, creando condiciones similares a las que existen en muchos lugares a lo largo del Eje Neovolcánico transversal de México en donde áreas severamente degradadas se encuentran adyacentes a bosques con diferentes grados de conservación. En el caso particular de los arenales, una vez eliminada la principal barrera, la profundidad de la capa de ceniza volcánica (Gómez-Romero et al., 2006, Lindig-Cisneros et al., 2006), es posible poner a prueba hipótesis relacionadas con el papel de la herbivoría, la distancia a las fuentes de propágulos y la creación de micrositios en el establecimiento de plántulas. El papel de los micrositios, sensu Harper et al. (1965), no puede ser ignorado debido a que la remoción de la ceniza volcánica por maquinaria pesada deja una capa de hasta $10 \mathrm{~cm}$ de arena en cerca del $90 \%$ de las áreas sometidas a la remoción, que aún puede representar una barrera para la germinación de las semillas y las etapas más incipientes de la vida de las plántulas. Una forma eficiente de crear micrositios en condiciones de restauración ecológica es agregar un acolchado que crea condiciones de sombra en el sustrato (Barradas, 1999).

Usando como modelo de estudio un arenal en donde se removió la ceniza volcánica en el paraje conocido como "Llano de Pario", en la comunidad de NSJP, Michoacán, se probaron las siguientes hipótesis: 1) La herbivoría limitará el establecimiento de plántulas tanto en cantidad como en términos de la composición de las comunidades de plántulas; 2) La distancia al borde del bosque (la fuente de propágulos) afectará el establecimiento de plántulas de tal forma que se encontrarán más plántulas cerca del borde, y 3) El uso de un acolchado creará condiciones que favorecerán al establecimiento de plántulas creando micrositios en donde las condiciones serán más favorables para el establecimiento de plántulas.

\section{Materiales y Métodos}

Diseño experimental de campo. Para poner a prueba las hipótesis planteadas en este estudio se estableció un experimento ortogonal para los factores exclusión de herbívoros y acolchado (micrositios) a diferentes distancias del borde del bosque del arenal conocido como "Llano de Pario" (19²9'6”N, 10241'46”O) en NSJP, Michoacán, México. Se establecieron 144 parcelas circulares con un área de $1 \mathrm{~m}^{2}$ cada una. Del total de parcelas, 72 fueron cercadas para protegerlas contra mamíferos herbívoros pequeños con malla de gallinero de $60 \mathrm{~cm}$ altura y enterrada $20 \mathrm{~cm}$, y $72 \mathrm{se}$ mantuvieron sin cercado contra herbívoros. Para evaluar el efecto de la variación de condiciones de los micrositios se establecieron tres tratamientos de acolchado agregando dos cantidades de paja de avena; $227 \mathrm{~g} / \mathrm{m}^{2}$ (baja) y $576 \mathrm{~g} / \mathrm{m}^{2}$ (alta) y un control. Se eligió paja porque un experimento anterior en otro arenal (Gil-Solórzano et al., 2009) sugirió que este material podía favorecer el establecimiento de plántulas al alterar las condiciones microclimáticas en las que se encuentran las semillas (Barradas, 1999).

Con el diseño antes descrito se contó con 24 repeticiones de cada combinación de tratamientos exclusión $\times$ acolchado. Cada una de las repeticiones por combinación de tratamientos se colocó a una distancia diferente del bosque, determinadas al azar desde el borde hacia el centro del arenal. Para determinar el efecto de los tratamientos en el establecimiento de plántulas se evaluó una vez por mes durante la temporada de lluvias del año 2008, en cada visita se registró el número de plántulas en cada parcela así como la especie, o se colectaron individuos para su posterior identificación, a la que pertenecían. 
Evaluación de la presencia de herbívoros. Para contar con información adicional sobre los herbívoros potenciales se utilizaron trampas de arena con cebo para atraer a mamíferos pequeños y registrar sus huellas. Aunque es posible que el cebo atraiga especies que de otra manera no se aventurarían fuera del bosque o de la cercanía del borde, se prefirió este método porque permite contar con repeticiones comparables. Se colocaron tres trampas cada tres filas del experimento, se colocó arena del sitio tamizada para colocarla en un área de $1 \mathrm{~m}^{2}$ en cada trampa y se colocó una mezcla de cebos: zanahoria picada, manzana molida, avena con vainilla y crema de cacahuate. El cebo se colocó en la tarde y se revisó en la mañana del día siguiente. El muestreo para evaluar la presencia de herbívoros se realizó a principios de la temporada de lluvias en el mes de junio (2009).

Experimentos en invernadero. Para determinar las especies cuyas semillas se encontraban en la paja y pudieran establecerse en el experimento de campo, que podrían causar un sesgo en los datos, se llevó acabo un ensayo en charolas de plástico $(60 \times 60 \times 5 \mathrm{~cm})$ con un medio de crecimiento obtenido de un proveedor local, estéril (creci-root ${ }^{\circledR}$ Uruapan, Michoacán) donde se colocó una capa de paja, procedente del material usado en campo muestreado al azar, con un peso promedio por metro cuadrado correspondiente al promedio de los dos tratamientos de campo $\left(401 \mathrm{~g} / \mathrm{m}^{2}\right)$. Las charolas fueron colocadas en una casa de sombra y fueron regadas hasta que ya no emergieran más plántulas en un período de cuatro meses.

Para evaluar el banco de semillas se tomaron muestras de suelo de las 24 filas del experimento de campo las cuales se mezclaron con creci-root ${ }^{\circledR}$ para posteriormente colocar la mezcla en charolas, esperar la germinación de las semillas y poder identificar las especies que se encuentran en el banco de semillas que tal vez por las condiciones generadas por los tratamientos experimentales no pudieron germinar en campo.

Análisis de datos. Los datos obtenidos del reclutamiento de plántulas se analizaron siguiendo dos estrategias. En primer lugar, se usó un análisis de varianza para establecer el efecto de los tratamientos de exclusión de herbvíboros, acolchado y distancia al borde del bosque sobre el número total de plántulas por tratamiento. Debido a que las dos primeras son factores fijos y la tercera un factor aleatorio se usó un modelo lineal generalizado seguido por una prueba de hipótesis con error tipo II (Crawley, 2007) en el paquete estadístico R (R Development Core Team, 2008). La relación entre el número de plántulas y la distancia al bosque se exploró por medio de regresión lineal con el mismo paquete estadístico. Para evaluar el efecto de los tratamientos antes descritos para cada especie se siguió el mismo procedimiento.

Debido a que se deseaba explorar si la distribución de las plántulas de cada especie presentaba un máximo en alguna distancia al borde del bosque y a partir de éste un decremento asintótico se ajusto un modelo utilizando la ecuación y = $\mathrm{a} \times \mathrm{e}^{-x / b}$, en la cual " $\mathrm{y}$ " representa el número de plántulas, "a" es la ordenada al origen, " $x$ " la distancia al borde del bosque y el parámetro " $b$ " establece el máximo de la curva, con el programa LabPlot 1.6.0 (Gerlach, 2007). Con el número de plántulas por especie en cada parcela se calcularon las distancias de Bray-Curtis para todas las parcelas que presentaron al menos una plántula y a partir de la matriz de distancias se realizó un análisis de agrupamiento usando la regla de agrupamiento de Ward (Manly, 2000). Para explorar la correlación entre la matriz de distancias de Bray-Curtis y la distancia al borde del bosque (b) se llevó a cabo una prueba de Mantel y con los tratamientos de exclusión de herbívoros y acolchado pruebas de ANOSIM. También se llevó a cabo un análisis de componentes principales (Everitt, 2007). Los análisis multivariados se llevaron a cabo con BiodiversityR (Kindy y Coe, 2005) y con el paquete vegan para R (Oksanen et al., 2009). Con los valores obtenidos para el índice de Shannon se hizo también un análisis de varianza y debido a que sólo la distancia al borde del bosque fue significativa, se hizo un análisis de regresión con el índice de Shannon como variable dependiente. En todos los casos se verificó que se cumplieran con los supuestos de los análisis estadísticos, los datos reportados son medias y errores estándar al menos que se indique de otra manera.

\section{Resultados}

Experimento de campo. En total se establecieron 20,011 plántulas en el experimento de campo (Cuadro 1), de las cuales 17,932 corresponden a 6 especies: Tagetes micrantha con 32\% del total, Crusea longiflora 23\%, Poaceae (1) 16\%, Muhlenbergia minutissima 7\%, Cyperus sp. (1) 7\% y Senecio salignus 4\% . El número de plántulas decreció en función de la distancia al borde del bosque (Figura 1), presentándose 19,580 plántulas a menos de 60 metros del borde, que representan el $98 \%$ del total. En las parcelas con exclusión de herbívoros se establecieron $110 \pm 30$ plántulas y en las parcelas sin exclusión $169 \pm 43$ plántulas. En función del tratamiento de acolchado los valores fueron para el control $119 \pm 38$ plántulas, baja $168 \pm 57$ plántulas y alta $129 \pm 40$ plántulas.

De las tres variables, la distancia al borde del bosque tuvo un efecto significativo en el número de plántulas lo que se reflejó en el análisis de regresión (núm. plántulas $=474$ $5.7 \mathrm{x}$, en donde "x" es la distancia al borde en metros, $r^{2}$ $\left.=0.32, F_{(1,142)}=68, P<0.00001\right)$. El análisis de varianza del experimento completo, considerando las interacciones entre factores, arrojó un resultado similar pues solamente la distancia al bosque fue significativa $\left(F_{(1,132)}=69, P<\right.$ $0.00001)$ y la interacción entre la distancia al bosque y la exclusión de herbívoros resultó marginalmente significativa $\left(F_{(1,142)}=3, P=0.08\right)$. Cuando se analizó el efecto de las 


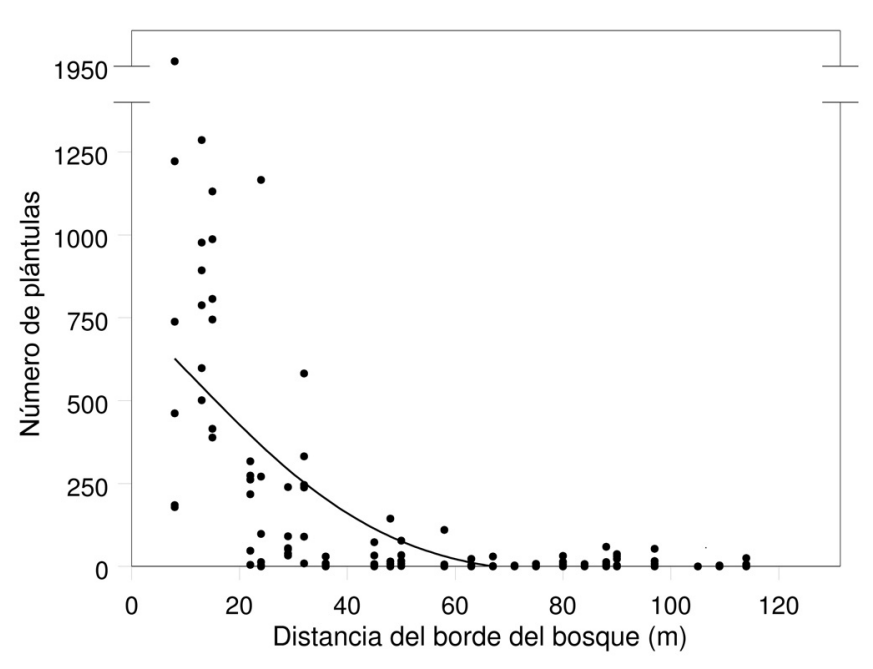

Figura 1. Número de plántulas en relación a la distancia del borde del bosque para todas las especies que se encontraron en las parcelas.

variables del experimento para las especies que presentaron suficientes plántulas para un análisis estadístico de forma individual (Cuadro 1), la variable más significativa para el establecimiento de la mayoría de las especies en las parcelas fue la distancia al borde del bosque, la cerca únicamente fue significativa para dos de las especies del género Cyperus. El efecto del acolchado (paja) fue significativo en interacción con las otras variables, una o ambas, para 4 especies (Cuadro 1), las cuales presentaron más plántulas en parcelas con acolchado que en las parcelas control, pero sólo para las parcelas que se encontraban en los primeros 60 metros a partir del borde del bosque, a distancias mayores el número de plántulas entre ambos tipos de parcelas fue muy similar.

De las especies que se establecieron en las parcelas y que presentaron suficientes individuos para ajustar una curva de la forma $\mathrm{y}=\mathrm{a} \times \mathrm{e}^{-x / b}, 11$ ajustaron de manera significativa $(P$ $<0.05)$ su abundancia en función a la distancia del borde y por lo tanto muestran un máximo en el número de plántulas a una distancia determinada (b): Asteraceae1 $(b=16 \mathrm{~m})$, Senecio salignus $(\mathrm{b}=5 \mathrm{~m})$ Tagetes micrantha $(\mathrm{b}=10 \mathrm{~m})$, Drymaria effusa $(\mathrm{b}=21)$, Cyperus $1(\mathrm{~b}=10 \mathrm{~m})$, Cyperus 2 $(\mathrm{b}=8 \mathrm{~m})$, Cyperus $3(\mathrm{~b}=9 \mathrm{~m})$, Phacelia platicarpa $(\mathrm{b}=18)$, Muhlembergia minutissima $(\mathrm{b}=7 \mathrm{~m})$ y Crusea longiflora $(\mathrm{b}$ $=6 \mathrm{~m}$ ).

Por otro lado, el análisis de agrupamiento de las distancias de la matriz de Bray-Curtis (Figura 2) indica que se forman 7 grupos con un $80 \%$ de similitud. Los grupos formados se caracterizan por encontrarse a diferentes distancias del borde del bosque. Tres grupos con distancias promedio de 14, $21 \mathrm{y}$ $46 \mathrm{~m}$ y 4 grupos, uno con una distancia promedio de $64 \mathrm{~m} \mathrm{y}$ los tres restantes con distancias promedio de $65 \mathrm{~m}$ del borde del bosque. Los tres grupos cuyas distancias promedio son

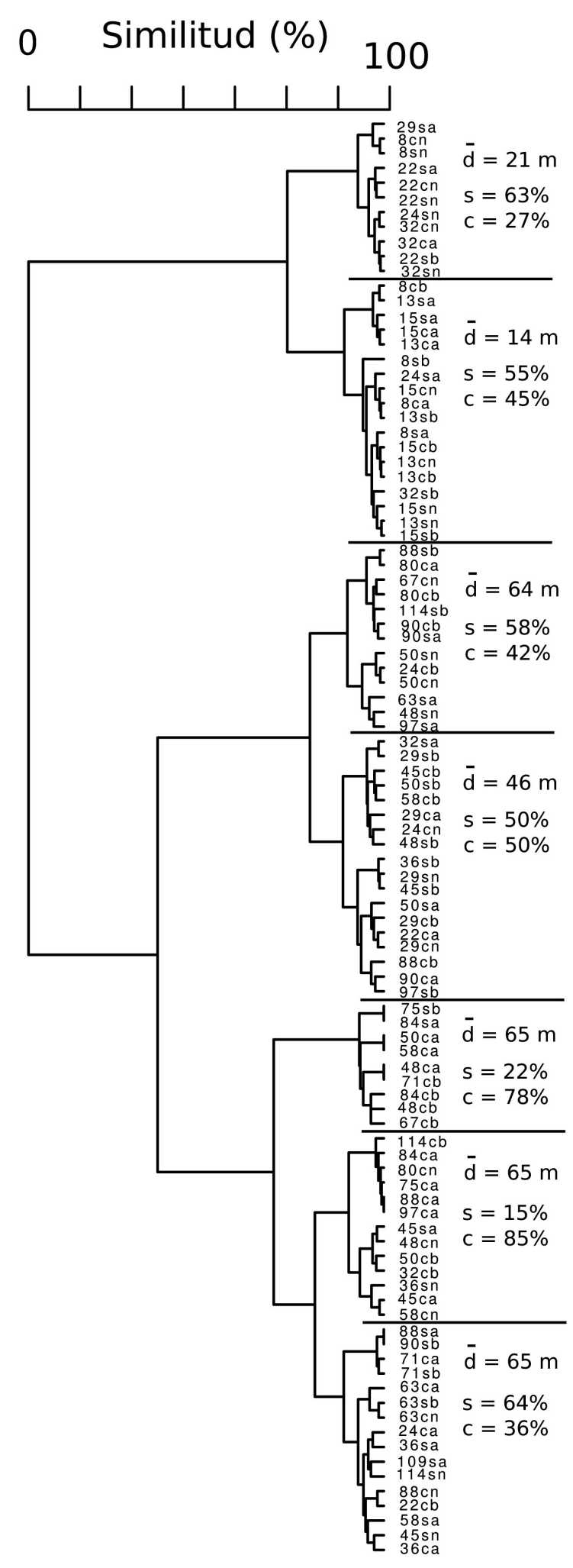

Figura 2. Agrupamiento de las parcelas de acuerdo a la distancia de Bray-Curtis y la regla de agrupamiento de Ward. Se forman 7 grupos con una simitud de $80 \%$ de los cuales se indica la distancia promedio al borde del bosque (d) y el porcentaje de parcelas con cercado (c) y sin cercado (s). 
LiMITACIONES PARA EL ESTABLECIMIENTO DE PLÁNTULAS EN ARENALES VOLCÁNICOS

Cuadro 1. Especies de las plántulas de las parcelas experimentales y resultados de los análisis de varianza para el número de plántulas por especie en respuesta a los tres factores del experimento de campo: distancia al borde del bosque, exclusión de herbívoros y acolchado. Se indica entre paréntesis la, interacciones significativa para cada especie $\left(n=\right.$ no significativo $\left.{ }^{*}<0.05,{ }^{* *}<0.01, * * *<0.001\right)$. Sólo se analizaron aquellas especies con números de muestra adecuados para el análisis.

\begin{tabular}{|c|c|c|c|c|c|c|}
\hline Familia & Especie & Plántulas & Distancia & Cerca & Paja & Interacción \\
\hline Asteraceae & Asteraceae 1 & 232 & $*$ & $\mathrm{n}$ & $\mathrm{n}$ & * (cerca:distancia) \\
\hline Asteraceae & Baccharis conferta Kunth & 7 & - & - & - & - \\
\hline Asteraceae & Conyza coronopifolia Kunth & 1 & - & - & - & - \\
\hline Asteraceae & Gnaphalium attenuatum DC & 20 & - & - & - & - \\
\hline Asteraceae & Heterotheca inuloides Cass. & 17 & - & - & - & - \\
\hline Asteraceae & Jaegeria hirta Less. & 15 & - & - & - & - \\
\hline Asteraceae & Senecio salignus DC & 904 & $* * *$ & $\mathrm{n}$ & $\mathrm{n}$ & $\begin{array}{l}* *(\text { distancia:paja }) \\
*(\text { cerca:distancia:paja })\end{array}$ \\
\hline Asteraceae & Tagetes micrantha Cav. & 6369 & $* * *$ & $\mathrm{n}$ & $\mathrm{n}$ & $\mathrm{n}$ \\
\hline Caryophyllaceae & Drymaria effusa A. Gray & 173 & $*$ & $\mathrm{n}$ & $\mathrm{n}$ & $\mathrm{n}$ \\
\hline Commelinaceae & Commelina tuberosa $\mathrm{L}$ & 1 & - & - & - & - \\
\hline Cyperaceae & Cyperus L 1 & 1480 & $* * *$ & * & $\mathrm{n}$ & $\begin{array}{l}\text { ** (cerca:distancia) } \\
* \text { (distancia:paja) }\end{array}$ \\
\hline Cyperaceae & Cyperus 2 & 105 & $* * *$ & * & $\mathrm{n}$ & * (cerca:distancia) \\
\hline Cyperaceae & Cyperus 3 & 126 & $\mathrm{n}$ & $\mathrm{n}$ & $\mathrm{n}$ & $\mathrm{n}$ \\
\hline Fabaceae & Dalea thouinii Schrank & 57 & $\mathrm{n}$ & $\mathrm{n}$ & $\mathrm{n}$ & $\begin{array}{l}* \text { (distancia:paja) } \\
* \text { (cerca:distancia:paja) }\end{array}$ \\
\hline Fabaceae & Trifolium repens $\mathrm{L}$ & 351 & $\mathrm{n}$ & $\mathrm{n}$ & $\mathrm{n}$ & $\mathrm{n}$ \\
\hline Guttiferae & Hypericum philonotis Cham. \& Schltdl. & 25 & - & - & - & - \\
\hline Hydrophyllaceae & Phacelia platycarpa Spreng. & 466 & $* *$ & $\mathrm{n}$ & $\mathrm{n}$ & $\mathrm{n}$ \\
\hline Labiatae & Prunella vulgaris $\mathrm{L}$ & 19 & - & - & - & - \\
\hline Onagraceae & Oenothera pubescens Ness. & 23 & - & - & - & - \\
\hline Pinaceae & Pinus pseudostrobus Lindl. & 2 & - & - & - & - \\
\hline Poacea & Poaceae 1 & 3191 & $* *$ & $\mathrm{n}$ & $\mathrm{n}$ & * (distancia:paja) \\
\hline Poaceae & Muhlenbergia minutissima (Steud.) Swallen & 1453 & $* * *$ & $\mathrm{n}$ & $\mathrm{n}$ & $\mathrm{n}$ \\
\hline Rosaceae & Alchemilla sibbaldiifolia Kunth & 8 & - & - & - & - \\
\hline Rubiaceae & $\begin{array}{l}\text { Crusea longiflora (Willd. ex Roem. \& Schult.) } \\
\text { W.R. Anderson }\end{array}$ & 4535 & $* * *$ & $\mathrm{n}$ & $\mathrm{n}$ & $\mathrm{n}$ \\
\hline Verbenaceae & Verbena bipinnatifida Schauer & 12 & - & - & - & - \\
\hline- & sp1 & 423 & $* *$ & $\mathrm{n}$ & $\mathrm{n}$ & $\mathrm{n}$ \\
\hline- & $\mathrm{sp} 2$ & 12 & - & - & - & - \\
\hline- & sp3 & 5 & - & - & - & - \\
\hline
\end{tabular}

iguales o menores a $46 \mathrm{~m}$ presentan un número muy similar de parcelas con exclusión y sin exclusión, lo que no ocurre en las parcelas cuya distancia promedio al borde es de 64-65 $\mathrm{m}$, para las cuales cada grupo muestra un porcentaje mucho mayor de parcelas de un tratamiento u otro. Estos patrones se reflejan en los valores de las pruebas de correlación entre la matríz de distancias de Bray-Curtis y la distancia al borde del bosque que fue significativo (estadístico de Mantel basado en 100 permutaciones: $r=0.7811, P=0.0099$ ), y la prueba para el efecto de la exclusión de herbívoros que fue marginalmente significativa (estadístico de ANOSIM basado en 100 permutaciones: $r=0.0223, P=0.0792$ ). El análisis de componentes principales indica que se forman dos grupos en relación a los dos primeros componentes (Figura
3), uno en el cual se agrupan la mayoría de las Poaceae y dos asteráceas, Senecio salignus y Tagetes micrantha, así como Crusea longiflora y Pinus pseudostrobus (Grupo A), y un segundo grupo con todas las demás especies. Estos dos grupos muestran una distribución diferente en función de la distancia al borde del bosque y en menor medida en función de la presencia de exclusiones (Figura 4). Las especies del grupo A tienden a estar presentes a distancias más cercanas al borde del bosque pero en menor proporción cuando hay exclusiones, las especies del grupo B se encuentran a todo lo largo del gradiente pero son más abundantes lejos del borde y no son tan afectadas por las exclusiones.

En términos de la diversidad por parcela, calculada como el índice de Shannon (Figura 5), sólo la distancia al borde 


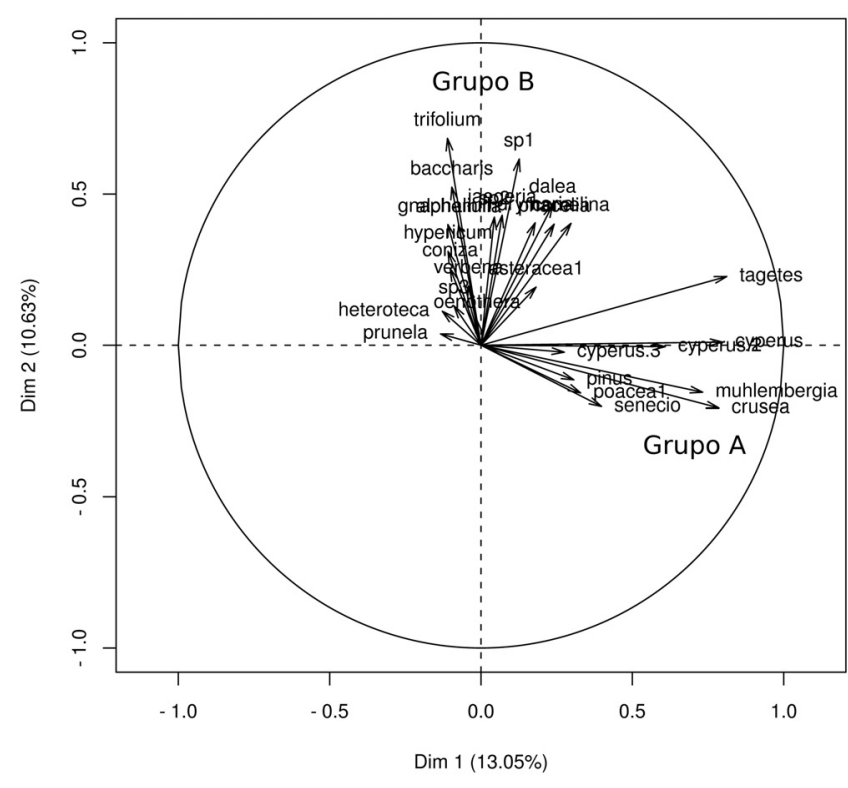

Figura 3. Mapa de las especies en función de los dos primeros componentes del análisis de componenetes principales.

de bosque fue significativa, pues este índice decrece en la medida en que la distancia aumenta, incluso excluyendo del análisis a todas las parcelas en donde el valor del índice fue cero $\left(r^{2}=0.3741, F_{(1,74)}=7.6, P<0.0072\right)$.

Evaluación de la presencia de herbívoros y experimentos en invernadero. El muestreo para evaluar la presencia de mamíferos al principio de la temporada de lluvias del 2009, mostró la presencia de huellas de conejos y roedores, aun-

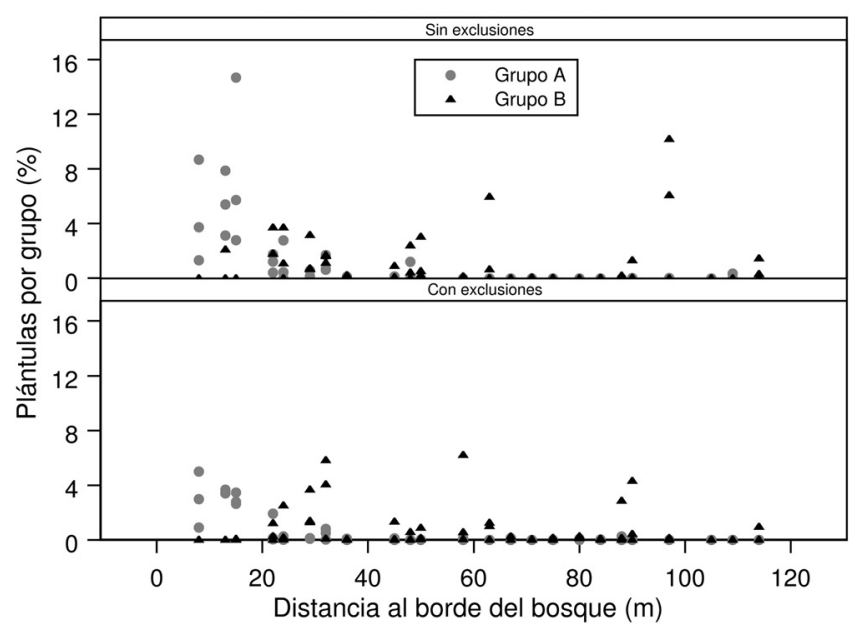

Figura 4. Proporción de las plántulas, en porcentaje, de cada uno de los grupos formados en el análisis de componentes principales. $\mathrm{La}$ distribución de las especies de cada grupo en función de la distancia al borde del bosque y de la presencia de exclusiones difiere.

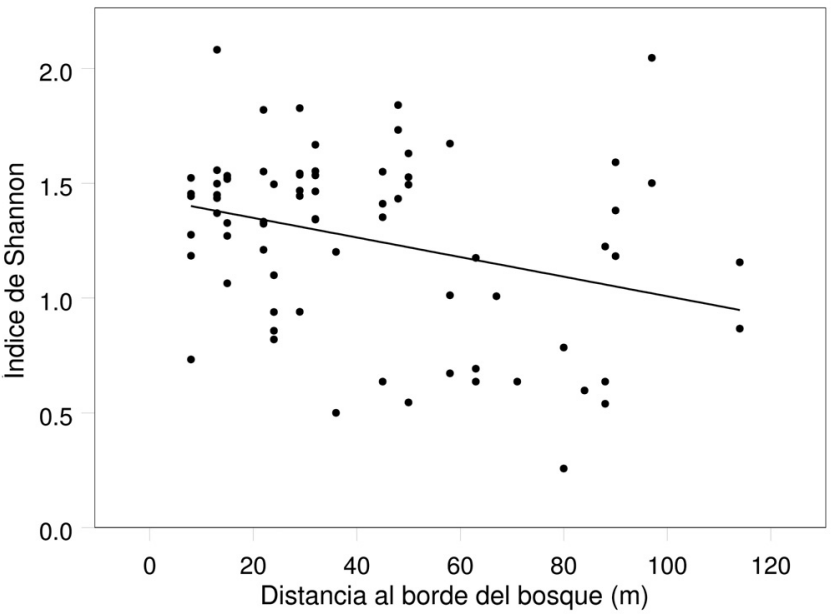

Figura 5. Relación entre el índice de Shannon de cada parcela y la distancia al borde del bosque, la relación entre ambas variables es significativa $\left(r^{2}=0.3741, F_{(1,74)}=7.6, P<0.0072\right)$.

que también se encontraron abundantes huellas de aves. En la zona más cercana al borde del bosque hasta una distancia de $24 \mathrm{~m}$ se encontraron huellas de los tres grupos de animales, de los 32 a los $50 \mathrm{~m}$ se encontraron huellas de roedores y aves; y de los $63 \mathrm{~m}$ en adelante sólo se encontraron huellas de aves.

Por otro lado, en las charolas en las cuales se colocaron muestras de la paja utilizada en campo para determinar si algunas de la especies que se encontraron en las parcelas pudieran derivarse de semillas que se encontraban en este material que se utilizó como acolchado, sólo se establecieron plantas de avena, la especie cultivada de la que se derivó la paja.

Finalmente, en el experimento de germinación del banco de semillas, las especies que se establecieron en el invernadero (Cuadro 2) corresponden a las mismas especies que se establecieron en el experimento en campo en el "Llano de Pario", por lo tanto, todas las especies encontradas en el banco de semillas pueden germinar en las condiciones del arenal y en las que se dan cuando se imponen los tratamientos experimentales.

\section{Discusión}

Los arenales de la comunidad de NSJP presentan condiciones adversas para el establecimiento de plántulas como por ejemplo la profundidad de la arena (Galindo-Vallejo, 2004; Gómez-Romero et al., 2006), temperaturas extremas (Blanco-García, 2005; Alvarado-Sosa, 2006), erosión pluvial (Blanco-García, 2005), falta de nutrientes (Gil-Solórzano et al., 2009) y la herbivoría en el caso del arbusto Lupinus elegans (Blanco-García y Lindig-Cisneros, 2005). En este estudio, las especies que se establecieron en las parcelas ex- 
Cuadro 2. Especies que se establecieron en invernadero de las muestras de suelo colectadas en campo mezcladas con el medio comercial de cultivo. Todas las especies que se establecieron en invernadero se establecieron en campo.

\begin{tabular}{ll}
\hline Familia Especie & \\
\hline Asteraceae 1 & Baccharis conferta \\
Asteraceae & Gnaphalium attenuatum \\
Asteraceae & Jaegeria hirta \\
Asteraceae & Senecio salignus \\
Asteraceae & Tagetes micrantha \\
Asteraceae & Drymaria effusa \\
Caryophyllaceae & Cyperus 1 \\
Cyperaceae & Dalea thouinii \\
Fabaceae & Trifolium repens \\
Fabaceae & Hypericum philonotis \\
Guttiferae & Phacelia platycarpa \\
Hydrophyllaceae & Prunella vulgaris \\
Labiatae & Oenothera pubescens \\
Onagraceae & \\
Poacea 1 & Muhlenbergia minutissima \\
Poacea & Alchemilla sibbaldiifolia \\
Rosaceae & Crusea longiflora \\
Rubiaceae & Verbena bipinnatifida \\
Verbenaceae & \\
\hline
\end{tabular}

perimentales corresponden, en su mayoría, a especies herbáceas, muchas de ellas anuales, que son dispersadas por viento. Para estas especies la variable más importante que explicó su distribución fue la distancia al borde del bosque, ya que el reclutamiento de plántulas disminuyó a medida en que esta distancia aumentaba. Este resultado sugiere que el establecimiento de plántulas en el arenal esta limitado por la dispersión de las díasporas (Matlack, 1994; Battaglia et al., 2002; Campbell et al., 2003), antes que ningún otro factor. Es interesante que 11 especies mostraron máximo número de plántulas por parcela a distancias entre los 5 y 15 metros del borde del bosque. Esto sugiere que son especies que se pueden establecer fuera de la influencia directa de los árboles del dosel del bosque pero que no encuentran condiciones propicias en las zonas más expuestas del arenal. Esto posiblemente se deba a las temperaturas extremas que se han identificado como uno de los factores que limitan el establecimiento de especies arbóreas en los arenales (BlancoGarcía, 2005; Alvarado-Sosa, 2006), A pesar de que en el sitio experimental se eliminó la mayoría de la arena, evitando así la barrera que capas gruesas de este material representa, aún permanecía una pequeña capa que podía alcanzar altas temperaturas. En este sentido, es interesante resaltar que el uso de paja como acolchado para crear micrositios para el establecimiento de plántulas (Barradas, 1999), no resultó significativo por si mismo y sólo fue importante para algunas especies (Cyperus sp, Senecio salignus, Dalea thouinii,
Poacea1 y sp. 2), a través de la interacción con la distancia al borde del bosque, la exclusión o ambas.

Los análisis de agrupamiento y de componentes principales arrojan información relevante para entender los factores limitantes para el establecimiento de plántulas. Por un lado, la composición de las comunidades de plántulas en las parcelas depende, al igual que el número de plántulas, principalmente de la distancia al borde del bosque. Esto se reflejó en que las distancias de obtenidas de la prueba Bray-Curtis se correlacionaran con la distancia al borde del bosque y marginalmente con la presencia de exclusiones de hervíboros. De acuerdo al análisis de componentes principales se formaron dos grupos de especies; el primero (grupo A) en donde se encuentran la mayoría de las especies de Poaceae y que se establecen cerca del borde del bosque y son afectados negativamente por las exclusiones, y el segundo (grupo B), formado por especies que se establecen a todo lo largo del gradiente y de manera abundante lejos del borde entre ellas las Asteráceas. Estos resultados indican que hay diferencias debidas a las estrategias de dispersión de las semillas.

Un aspecto importante de considerar fue el papel de la exclusión de mamíferos pequeños, potencialmente herbívoros, en el establecimiento de plántulas, ya que se conoce que las plantas son más susceptibles a la herbivoría en etapas tempranas (Carson y Root, 1999; Rodríguez de la Vega, 2003; Blanco-García, 2005; Howe, 2008). Sin embargo, en el experimento de campo se presentaron menos plántulas en las parcelas con exclusiones y por lo tanto el efecto fue el contrario al esperado. Este patrón sólo fue significativo para una de las especies del género Cyperus y marginalmente significativo para el conjunto de las plántulas. Este resultado puede ser consecuencia de que las semillas de la mayoría de las especies que se establecieron en número considerable son dispersadas por el viento, y por lo tanto las mallas de las exclusiones pudieron actuar como barreras. Sin embargo, en el caso de Senecio salignus, que pertenece al grupo A fue abundante cuando no hay exclusiones. Esta es una especie poco palatable, como otras del género (Caño et al., 2009) y se podría ver beneficiada por menor competencia causada por la remoción de otras especies por los herbívoros (Hanley, 2004). Esto es más probable que ocurra cerca del borde del bosque en donde se aventuran más tipos de animales de acuerdo a los datos arrojados por las trampas de cebo.

Por otro lado, se establecieron más plántulas en los tratamientos con acolchado que en el tratamiento control en general,aunque la diferencia fue significativa sólo en interacción con la distancia al borde del bosque, pues a distancias menores no había diferencias entre el número de plántulas con acolchado y sin el, pero a distancias mayores las hubo. Este efecto del acolchado es similar al observado en un experimento previo (Gil-Solórzano et al., 2009), en donde en parcelas con acolchado se presentaron más plántulas, sin que la diferencia fuera estadísticamente significativa debido al tamaño de muestra, aunque en ese estudio no fue 
posible determinar el efecto de la distancia al borde del bosque y de las exclusiones.

Los resultados obtenidos sugieren que la dispersión es el factor principal que limita el desarrollo de la vegetación en los arenales, debido a que la distancia al borde del bosque fue el factor más importante para explicar los patrones observados. Las consecuencias para la restauración de sitios que se encuentren severamente degradados son claras, pues si se desea contar con especies herbáceas en el sotobosque una vez que se logre una cobertura arbórea por reforestación, se tendría que esperar un tiempo considerable para que se establezcan debido a que la colonización procederá desde el borde del bosque lentamente, o habría que introducirlas como parte del esfuerzo de restauración.

\section{Agradecimientos}

Este proyecto fue financiado por CONACyT (SEMARNAT)-2002-C01-0760 a RLC. Se agradece a la Dra. Yvonne Herrerías Diego y al Dr. Juan Carlos Montero Castro, así como a dos revisores anónimos por sus comentarios y observaciones al trabajo. A la CINSJP por su apoyo para realizar esta investigación.

\section{Literatura citada}

Alvarado-Sosa P.A. 2006. Ensayo de especies y de coberturas para restaurar sitios con distinto grado de disturbio. Tesis de Maestría, Facultad de Biología, Universidad Michoacana de San Nicolás de Hidalgo, Morelia, 86 pp.

Barradas V.L. 1999. Ecofisiología vegetal: una herramienta para la conservación y restauración ecológica. Boletín de la Sociedad Botánica de México 65:71-72.

Battaglia L.L., Minchin P.R. y Pritchett D.W. 2002. Sixteen years of old-field succession and reestablishment of a bottomland hardwood forest in the lower Mississippi alluvial valley. Wetlands 22:1-17.

Blanco-García J.A. 2005. Efecto del esfuerzo de restauración de la vegetación nativa aledaña al volcán Paricutín, Michoacán, México. Tesis de maestría. Posgrado en Ciencias Biológicas. Universidad Nacional Autónoma de México, México D.F. 53pp.

Blanco-García A. y Lindig-Cisneros R. 2005 Incorporating restoration in sustainable forestry management: Using pine bark mulch to improve native species establishment on tephra deposits. Restoration Ecology 13:703-709.

Bonfil C. 1998. The effects of seed size, cotyledon reserves, and herbivory on seedling survival and growth in Quercus rugosa and Q. laurina (Fagaceae). American Journal of Botany 85:7987

Bonfil S.C., Rodríguez de la Vega H. y Peña V. 2000. Evaluación del efecto de las plantas nodrizas en el establecimiento de una plantación de Quercus L. Ciencia Forestal en México 25:5973.

Campbell D.R., Rochefort L. y Lavoie C. 2003. Determining the immigration potential of plants colonizing disturbed environments: the case of milled peatlands in Quebec. Journal of
Applied Ecology 40:78-91.

Caño L., Escarré J., Vrieling K. y Sans F.X. 2009. Palatability to a generalist herbivore, defense and growth of invasive and native Senecio species: testing the evolution of increased competitive ability hypothesis. Oecologia 159:95-106.

Carson W.P. y Root R.B. 1999. Top-down effects of insect herbivores during early succession: influence on biomass and plant dominance. Oecologia 121:260-272.

Case R.L. y Kauffman J.B. 1997. Wild ungulate influences on the recovery of willows, black cottonwood, and thin-leaf alder following cessation of cattle grazing in northeast Oregon. Northwest Science 71:115-126.

Delgado-Granados, H. 2001. Los volcanes de México. Universidad Nacional Autónoma de México. 227p.

Crawley M.J. 2007. The R book. John Wiley and Sons, Ltd., West Sussex, England.

del-Val E., Armesto J.J., Barbosa O. y Marquet P.A. 2007. Effects of herbivory and patch size on tree seedling survivorship in a fog-dependent coastal rain forest in semiarid Chile. Oecologia 153:625-632.

del-Val E. y Crawley M.J. 2005. What limits herb biomass in grasslands: competition or herbivory. Oecologia. 142:202-211.

Eggler W.A. 1959. Manner of invasion of volcanic deposits with further evidence from Paricutín and Jorullo. Ecological Monographs 29:267-284.

Eggler W.A. 1963. Plant life of Paricutín volcano, México, eight years after activity ceased. American Midland Naturalist 69:3868.

Everitt B. 2007. An R and S-plus companion to multivariate analysis. Springer. London.

Frankland F. y Nelson T. 2003. Impacts of white-tailed deer on spring wildflowers in Illinois, USA. Natural Areas Journal 23:341-348.

Galindo-Vallejo S. 2004. Estudio de la vegetación nativa asociada a Los depósitos de ceniza volcánica en la comunidad de Nuevo San Juan Parangaricutiro, Michoacán México. Tesis de licenciatura. Facultad de Biología. Universidad Michoacana de San Nicolás de Hidalgo. México. 44pp

Gerlach S. 2007. Labplot 1.6.0: An application for plotting and analysis of 2D and 3D functions and data. <http://labplot.sourceforge.net> Febrero, 2010.

Gil-Solórzano D., Lara-Cabrera S. y Lindig-Cisneros R. 2009. Effects of organic matter added to sand deposits of volcanic origin on recruitment of seedling. Southwestern Naturalist 54:439-445.

Gómez-Romero M., Lindig-Cisneros R. y Galindo-Vallejo S. 2006. Effect of tephra depth on vegetation development in areas affected by volcanism. Plant Ecology 183:207-213.

Hanley M.E. 2004. Seedling herbivory and the influence of plant species richness in seedling neighbourhoods. Plant Ecology 170:35-41.

Harper J.L., Williams J.T. y Sagar G.R. 1965. The behavior of seeds in soil. I. The heterogeneity of soil surfaces and its role in determining the establishment of plants from seed. Journal of Ecology 53:273-286.

Holl K.D. y Quiros-Nietzen E. 1999. The effect of rabbit herbivory on reforestation of abandoned pasture in southern Costa Rica. Biological Conservation 87:391-395.

Howe H.F. 2008. Reversal of fortune: plant suppression and recovery after vole herbivory. Oecologia 157:279-286. 
Kindt R. y Coe R. 2005. Tree diversity analysis. A manual and software for common statistical methods for ecological and biodiversity studies. World Agroforestry Centre (ICRAF), Nairobi, Kenia.

Lindig-Cisneros R., Galindo-Vallejo S. y Lara-Cabrera S. 2006. Vegetation of Thepra deposits 50 years after the end of the eruption of the Paricutín volcano, México. Southwestern Naturalist 51:455-461.

Manly B.F.J. 2000. Multivariate statistical methods: a primer. Chapman and Hall, Boca Raton, Florida.

Matlack G.R. 1994. Plant species migration in a mixed-history forest landscape in eastern North America. Ecology 75:14911502.

Oksanen J., Kindt R., Legendre P., O’Hara B., Simpson G.L., Solymos P., Henry M., Stevens H. y Wagner H. 2009. vegan: Community Ecology Package. R package version 1.15-4. http://CRAN.R-project.org/package=vegan

Opperman J.J. y Merenlender A.M. 2000. Deer Herbivory as an Ecological Constraint to Restoration of Degraded Riparian Co- rridors. Restoration Ecology 8:41-47.

R Development Core Team, 2008. R: A Language and Environment for Statistical Computing. R Foundation for Statistical Computing, Vienna, Austria. <www.R-project.org>. Febrero 2010.

Rejmánek M., Haagerova R. y Haager J. 1982. Progress of plant succession on the Paricutin volcano- 25 years after activity ceased. American Midland Naturalist 108:194-198.

Rodríguez-de la Vega H.F. 2003. Estructura poblacional y distribución espacial de Senecio praecox en el Ajusco medio, D.F., implicaciones para su reintroducción en sitios perturbados. Tesis de licenciatura.Universidad Nacional Autónoma de México, México D.F., 78pp.

Shelton A.L. y Inouye R.S. 1995. Effect of browsing by deer on the growth and reproductive success of Lactuca Canadensis (Asteraceae). American Midland Naturalist 134:332-339.

Sweeney B.W., Czapka S.J. y Yerkes T. 2002. Riparian Forest Restoration: Increasing Success by Reducing Plant Competition and Herbivory. Restoration Ecology 10:392-400. 